\title{
Pulmonary hemosiderosis with normocomplementemic urticarial vasculitis in a child
}

\author{
H. Yuksel1 ${ }^{1}$ O. Yilmaz¹, R. Savas², C. Kirmaz³ , A. Sogut ${ }^{1}$, S. Özalp4
}

ABSTRACT: Pulmonary hemosiderosis with normocomplementemic urticarial vasculitis in a child. H. Yuksel, O. Yilmaz, R. Savas, C. Kirmaz, A. Sogut, S. Özalp.

Pulmonary hemosiderosis is rarely associated with urticarial vaculitis especially if normocomplementemic. An eigth year old girl presented with relapsing and remitting chronic and persistent urticarial lesions, conjunctival injection, recurrent cough and hemoptysis. Respiratory findings started at seven years of age. Physical examination revealed diffuse skin lesions mainly settled on the extremites, nonpurulent conjunctival injection, rare ronchi and fine crackles in bilateral lower zones of the lungs. Biopsy of the urticaria like skin lesions demonstrated leukocytoclastic vasculitis. Rheumatological markers were negative. Levels of complement fractions 3 and 4 were normal. Chest $x$-ray demonstrated diffuse alveolar infiltrative images. High Resolution Computed Tomography of the chest revealed diffuse ground-glass appearance, in- creased interstitial density. Diagnostic flexible fiberoptic bronchoscopy was performed and bronchoalveolar lavage fluid revealed hemosiderin laden alveolar macrophages. She was started on systemic corticosteroid treatment. During follow up, pulmonary symptoms disappeared, however skin lesions and conjunctival symptoms persisted and exacerbated four times in two years. CT of lungs after two years of treatment revealed rare patchy areas of ground glass appearance in bilateral lower lobes and right upper lobe as well as a few of milimetric pleural nodules. This patient is still followed up under low dose steroids and pulmonary findings regressed but low grade inflammation due to vasculitis is thought to continue as supported by the persistence of tomographic findings in the lungs despite the absence of any symptoms. This case demonstrates association of urticarial vasculitis and pulmonary hemosiderosis in the setting of normocomplementemia. Monaldi Arch Chest Dis 2007; 67: 1, 63-66.

Keywords: Pulmonary hemosiderosis, urticaria, vasculitis, children.

1 Celal Bayar Universiy, Dep. of Pediatric Allergy and Pulmonology, Manisa,

2 Ege University, Dep. of Radiology, Manisa,

3 Celal Bayar University, Dep. of Immunology and Allergy, Manisa,

4 Tepecik State Hospital, Dep. of Pediatrics, Izmir, Turkey.

Correspondance: Ozge Yulmaz, MD; 858 sokak Cakiroglu ish No 2/304 Konak, 35250 Izmir, Turkey; e-mail: oyilmaz_76@hotmail.com

\section{Introduction}

Pulmonary hemosiderosis $(\mathrm{PH})$ is a term used to define an abnormal accumulation of hemosiderin in the lung parenchyma secondary to alveolar haemorrhage [1]. Alveolar hemorrhage in this entity can have various etiologies such as cardiac disease leading to increased pulmonary venous hypertension, bleeding diathesis, systemic vasculitis syndromes and primary lung disorders [1]. The main pathophysiological point is an alveolo-capillary bleeding into the alveolar space and perialveolar zone $[1,2]$. Theoretically, alveolar blood is absorbed by alveolar macrophage or is expectorated. Perialveolar hemorrhage leads to interstitial degenerative and inflammatory reactions [3]. Therefore, this syndrome usually presents with hemoptysis, diffuse parenchymal infiltrates in chest radiographs, iron deficiency anemia and rare symptoms like hematuria, splenomegaly. Many paediatric cases were diag- nosed as having an idiopathic origin in previous years [4]. However, the number of idiopathic cases (IPH) has gradually decreased by definition and diagnosis of vasculitic syndromes [1, 3-5]. One of the rarely reported causes is urticarial vaculitis (UV) characteriaed by persistent urticarial lesions that are associated with leukocytoclastic vasculitis of dermal vessels $[4,6]$. However association with $\mathrm{PH}$ is reported in a very few cases in literature which were reported to be hypocomplementemic type [7]. No case report about a normocomplementemic case could be found. Conjunctivitis without evidence of uveitis was reported to coexist with UV, too [7].

The case presented in this report was diagnosed to have normocomplementemic UV associated with conjunctivitis at three years of age and developed PH at the age of seven years. This case was reported to emphasize that pulmonary hemosiderosis is a possibility in UV cases even if hypocomplementemia is not present. 


\section{Case report}

An eight year old girl presented with chronic and persistent urticarial lesions, conjunctival injection, recurrent cough and hemoptysis. Non-itchy urticarial lesions distributed all over the body were present since the age of three years. These lesions that followed a pattern of exacerbations and remissions sometimes appeared like areas of bleeding in skin and also coincided with exacerbated conjunctival injection. Respiratory findings started at seven years of age as periods of cough and hemoptysis. Since hemoptysis was not massive, and computed tomography (CT) of the chest of the lungs interpreted to be normal except for the subsegmental athelectasis of the right middle lobe, she was thought to have asthma and inhaled corticosteroids were initiated. She presented to our clinic at eight years of age upon persistence of pulmonary findings despite this treatment. It was reported that urticarial lesions and conjunctival injection continued to wax and wane throughout this period. Past history was unremarkable as well as the family history. Physical examination revealed diffuse skin lesions mainly settled on the extremities, non-purulent conjunctival injection, rare ronchi and fine crackles in bilateral lower zones of the lungs. Vasculitic characteristics of these urticarial lesions were absence of itching, failure to fade with pressure, being rounded and induration. Complete blood count was normal at presentation with $\mathrm{Hb}$ of $11.6 \mathrm{~g} / \mathrm{dL}$ Htc of $35.5 \% \mathrm{MCV}$ of $80 \mathrm{Fl}$ thrombocytes of $420000 / \mathrm{mm}^{3}$. However, it was reported by the family that she had anaemia and had received iron treatment during her initial presentation to the first hospital. Iron and ferritin levels were $42 \mu / \mathrm{dL}$ and $56 \mathrm{ng} / \mathrm{mL}$ respectively. Biochemical parameters including renal and liver function tests, hemorragic diathesis tests, such as prothrombin time etc were in normal ranges. Mantoux test was normal. Biopsy of the urticaria like skin lesions demonstrated significant neutrophilic infiltration including eosinophils prominent around the vessels in superficial dermis. Neutrophils were encountered in vessel walls and lumen (fig. 1). PAS and elastic staining revealed disruption of vessel wall continuity

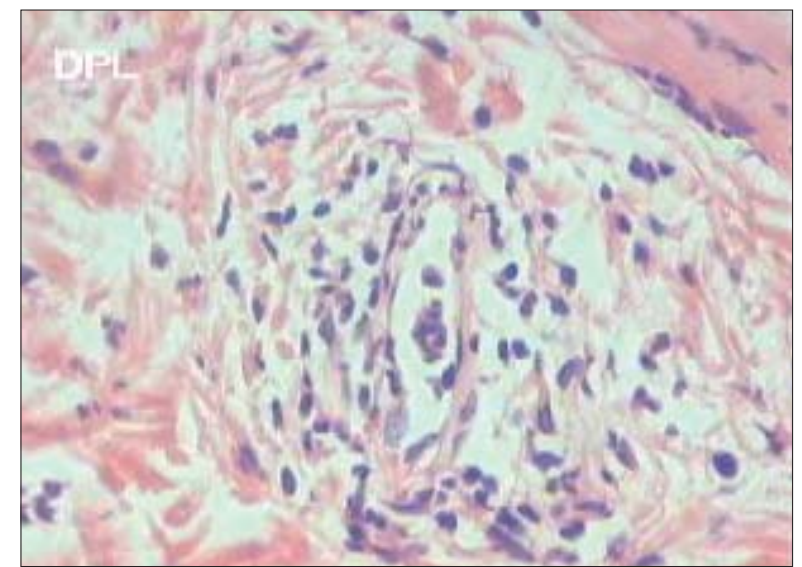

Fig. 1. - Biopsy of the urticarial lesion demonstrated lymphocyte and eosinophil accumulation in perivascular area and vessel wall, erythrocyte extravasation and fibrin deposition. and red blood cell extravasation. Fibrinoid necrosis was evident. Direct immunofluorescent examination did not show immune deposition. These findings of leukocytoclastic vasculitis were interpreted in favor of urticarial vasculitis. Rheumatological markers including double-strain-antinuclear antibody, p- and c- antinuclear cytoplasmic antibody, rheumatoid factor, anti-threoglobuline, anti-microsomal antibodies were all negative. Levels of complement-3 and 4 were found to be in normal ranges. The patient was not able to perform spirometry. Serum immunoglobulin-A, G, M, E levels were normal. Serum cows' milk specific immunoglobulin-E and skin prick test were negative. Total complement and other complement fractions were not determined.

Chest-x-ray demonstrated diffuse alveolar infiltrative images. High Resolution Computed Tomography (HRCT) of the chest revealed diffuse ground-glass appearance, increased interstitial density (fig. 2 A, B). These findings were consistent with pulmonary hemosiderosis therefore diagnostic flexible fiberoptic bronchoscopy was performed and microscopic examination of bronchoalveolar lavage fluid revealed hemosiderin laden alveolar macrophages specific for pulmonary hemosiderosis (fig. 3).

She was started on systemic corticosteroid treatment with prednisolone at a dose of 2 $\mathrm{mg} / \mathrm{kg} /$ day. Clinical response of pulmonary findings was improved dramatically. Skin lesions and conjunctivitis decreased significantly but did not
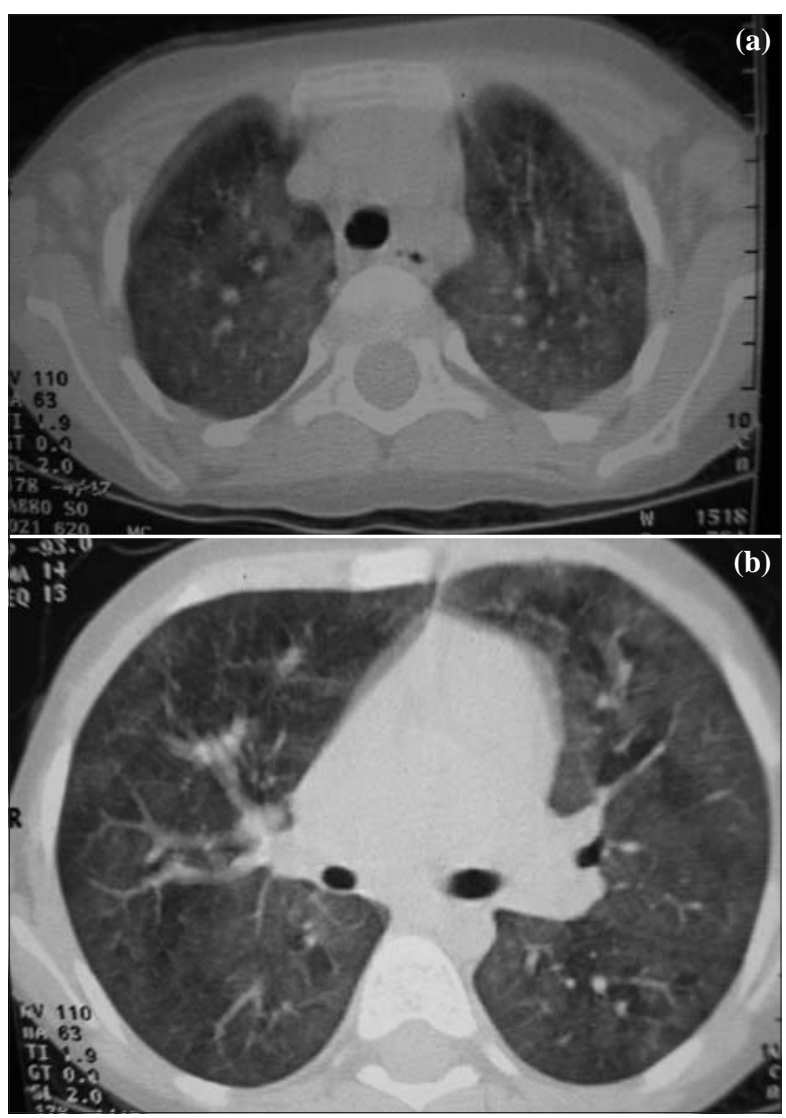

Fig. 2. - CT showed bilaterally increased density of upper lobes (a) and lower lobes (b) of pulmonary parenchyma as signs of diffuse alevolar hemorrhage. 


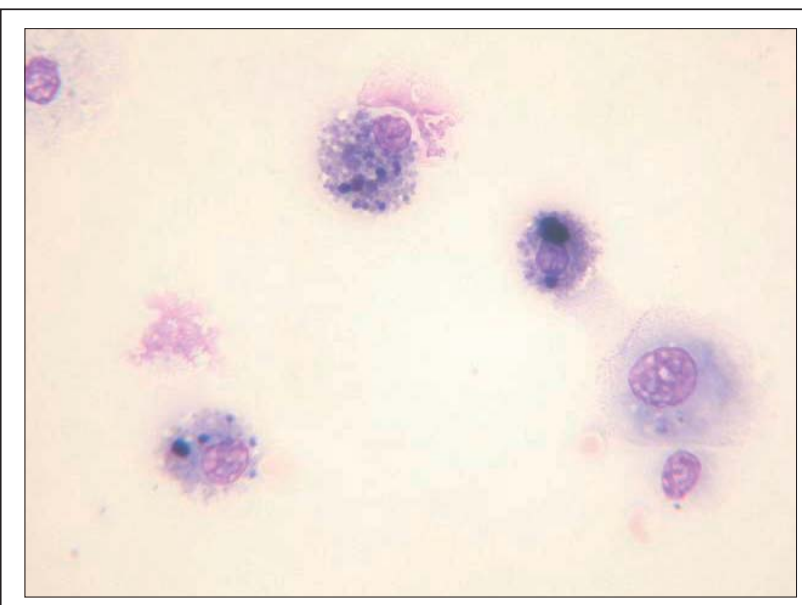

Fig. 3. - Hemosiderin-laden macrophages were demonstrated in bronchoalveolar lavage fluid.

disappear completely. Oral corticosteroid treatment was tapered gradually and continued at a dose of $10 \mathrm{mg}$ alternate day for two years. During follow up, pulmonary symptoms disappeared, however skin lesions and conjunctival symptoms persisted and exacerbated four times in two years. Interestingly, exacerbation of skin lesions accompanied increased conjunctival injection. CT of lungs after two years of treatment revealed rare patchy areas of ground glass appearance with vague borders in bilateral lower lobe superior segments and right upper lobe. A few of millimetric pleural nodules were noted in the posterior pleura neighboring the right lower lobe superior and posterior basal segments. These cortical interstitial changes observed in lung parenchyma were consistent with pulmonary hemosiderosis.

This patient is still followed up under low dose steroids and pulmonary findings regressed but low grade inflammation due to vasculitis is thought to continue as supported by the continuance of tomographic findings in the lungs despite absence of symptoms.

\section{Discussion}

Pulmonary hemosiderosis that is defined as the abnormal accumulation of hemosiderin in the interstitium of lungs due to diffuse alveolar hemorrhage is an uncommon pulmonary problem in childhood $[1,5]$. Pulmonary hemorrhage in lungs leads to ingestion of haemoglobin by macrophages in which it is converted to hemosiderin by lysosomal degradation [8]. Pulmonary hemosiderosis may be primary or secondary to many systemic diseases including vasculitis syndromes like polyarteritis nodosa, Wegener syndrome, systemic lupus erythematosus, and rheumatoid arthritis [9]. Episodes of pulmonary haemorrhage associated with diffuse pulmonary infiltrates and iron deficiency anemia is defined as primary pulmonary hemosiderosis $[1,8$, 9]. It has been postulated that childhood hemosiderosis is mostly not idiopathic and may precede more systemic diseases due to the immunological nature of its pathogenesis [10]. Although, many paediatric cases were diagnosed as having an idiopathic origin before, the number of idiopathic cas- es (IPH) has gradually decreased in the last years with the definition and diagnosis of vasculitic syndromes affecting pulmonary parenchyme such as systemic lupus erythematosus, Churg-Strausse syndrome, Panarteritis nodosa etc. [8]. Moreover, many other etiological factors may rarely have a role in the pathogenesis of pulmonary hemosiderosis such as cardiovascular disorders that had led to increased pulmonary venous pressure (cor-triatriatum, mitral stenosis etc), systemic haemorrhagic diathesis (thrombocyte disorders, hemophilia syndrome etc) and others $[1,2,8,9]$. This data demonstrates that a possible primary etiological factor, especially vasculitis syndromes, should be searched for in all children with pulmonary hemosiderosis. Therefore, these cases can be classified as idiopathic pulmonary hemosiderosis only if a vasculitis syndrome or another primary cause (cardiovascular disorders or etc) are not detected [8]. When etiology is considered, our case presents a rare condition because of the fact that an association of pulmonary hemosiderosis with normocomplementemic urticarial vasculitis has not been reported previously. This type of urticarial vasculitis has not been reported as an etiological factor for pulmonary hemosiderosis in the cases reported previously.

Clinically pulmonary hemosiderosis is characterised by the triad of iron deficiency anemia, diffuse pulmonary infiltrates and chronic pulmonary symptoms [1, 2, 8]. Our case displayed all the characteristic findings including recurrent episodes of cough and hemoptysis as well as the typical chest-X-ray and HRCT findings. As has been stated in previous literature a precise diagnosis was achieved through microscopic examination of bronchoalveolar lavage fluid that demonstrated hemosiderin laden alveolar macrophages [1]. The disease is characterised by cycles of clinical remission, pre-acute and acute phases of pulmonary bleeding [11]. The episodes of cough and hemoptysis that followed a course of relapses and remissions in our case were similar to the previously reported cases [11]. Demonstration of increased neutrophils in bronchoalveolar lavage fluid even during remission as reported before is important in evaluation of treatment in these patients [11]. In our case, clinical findings of pulmonary disease regressed with long term low dose steroid treatment. However, the findings of ground glass appearance, pleural nodules indicating cortical interstitial changes even at second year of treatment without pulmonary symptoms suggested that inflammation due to minimally active vasculitis may be ongoing without an active hemorrhage/hemoptysis in addition to the chronic changes including fibrous scars. It may be suggested that the follow up of children with pulmonary hemosiderosis should consider not only clinical symptoms but also HRCT findings.

Prognosis of pulmonary hemosiderosis has been reported to be associated with many findings, none of which is sure. Initial fever, leukocytosis, hepatosplenomegaly were clinical findings reported to be associated with poor prognosis [10]. Similarly, presence of autoantibodies especially AN$\mathrm{CA}$ was reported to indicate poor prognosis [10]. 
Rhematological factors including antinuclear antibody, p- and c- antinuclear cytoplasmic antibodies were absent in our case [10]. Additionally, Heiner syndrome, an association of pulmonary hemosiderosis and cows' milk hypersensitivity, has a good prognosis after cessation of oral intake of cow milk [1, 3]. However cows' milk hypersensitivity was absent in our case because serum cows' milk specific IgE and skin prick test with cows' milk allergen extract were negative.

The clinical spectrum of the case described in this article has a different aspect because the disease was initiated with chronic urticarial lesions diagnosed as urticarial vasculitis that preceded the development of pulmonary findings by four years. Urticarial vasculitis that is characterised by urticarial lesions persisting more than 24 hours and leukocytoclastic vasculitis of dermal vessels was diagnosed in this case by skin biopsy findings that demonstrated perivascular neutrophilic infiltration and fibrinoid necrosis [6]. This clinico-pathological condition may be associated with normal or decreased complement levels $[6,12]$. It is thought to be due to type 3 hypersensitivity reaction because circulating immune-complexes have been encountered in $30-75 \%$ of patients and these are thought to deposit in blood vessel walls and activated complement cascade [13]. Urticarial vasculitis may be associated with connective tissue diseases especially systemic lupus erythematosus [6]. In only one case has pulmonary disease been previously reported to be associated with hypocomplementemic urticarial vasculitis $[7,12,13]$. Obstructive airway disease and asthma has been reported in 5-20\% of cases [13]. In our case, normocomplementemic urticarial vasculitis precedes pulmonary findings by four years. There are no paediatric cases in literature to our knowledge that reported pulmonary hemosiderosis in normocomplementemic urticarial vasculitis.

Pathogenesis of urticarial vasculitis enlightens its association with pulmonary haemorrhage and hemosiderosis. Immunoreactants are seen to deposit around blood vessels and basement membrane leading to postcapillary venulitis that is characterized by endothelial necrosis and perivascular inflammatory cellular infiltration [13]. In many secondary cases of pulmonary hemosiderosis like Goodpasture's syndrome or Heiner's syndrome immunological mechanisms leading to basement membrane and endothelial damage have been found [8)]. Idiopathic pulmonary hemosiderosis is also thought to be an immunological disease resulting in a possible defect in the basement membrane of the pulmonary capillaries [4]. The primary defect was postulated not to be in alveoli but in circulation due to the increase in immune complexes which activate complement cascade and induce release of mediators that lead to polymorphonuclear accumulation and degranulation leading to vascular damage [11]. These immunological mechanisms postulated make the association of urticarial vasculitis with pulmonary hemosiderosis possible. However, the absence of hypocomplementemia is interesting in this case because these immunological mechanisms of immune complex deposition would be expected to lead to hypocomplementemia.

Pulmonary hemosiderosis is named to be idiopathic in decreasing number of patients with time and this is expected to decrease further as the underlying pathogenetic mechanisms are understood better. This case demonstrates association of urticarial vasculitis and pulmonary hemosiderosis in the setting of normocomplementemia. This finding may be important to delineate further research about the immunological mechanisms leading to vascular damage in this disease.

\section{References}

1. Yao TC, Hung IJ, Wong KS, Huang JL, Niu CK. Idiopathic pulmonary haemosiderosis: an Oriental experience. J Paediatr Child Health 2003; 39: 27-30.

2. Yao TC, Hung IJ, Jaing TH, Yang CP. Pitfalls in the diagnosis of idiopathic pulmonary haemosiderosis. Arch Dis Child 2002; 86: 436-8.

3. Saeed MM, Woo MS, MacLaughlin EF, Margetis MF, Keens TG. Prognosis in pediatric idiopathic pulmonary hemosiderosis. Chest 1999; 116: 721-5.

4. Paul G, Bhatnagar SK, al Maskary S, Kuruvilla S, Kurien S. Idiopathic pulmonary haemosiderosis. J Trop Pediatr 2000; 46: 243-5.

5. Godfrey S. Pulmonary hemorrhage/hemoptysis in children. Pediatr Pulmonol 2004; 37: 476-84.

6. Soylu A, Kavukcu S, Uzuner N, Olgac N, Karaman O, Ozer E. Systemic lupus erythematosus presenting with normocomplementemic urticarial vasculitis in a 4-yearold girl. Pediatr Int 2001; 43: 420-2.

7. Al Riyami BM, Al Kaabi JK, Elagib EM, Khatim HS, Woodhouse NJ. Subclinical pulmonary haemorrhage causing a restrictive lung defect in three siblings with a unique urticarial vasculitis syndrome. Clin Rheumatol 2003; 22: 309-13.

8. Mc Coy KS. Hemosiderosis. In: Taussig LM, Landau LI, eds. Pediatric Respiratory Medicine. 1st ed. United States of America: Mosby; 1999: 835-841.

9. Chu SH, Shyur SD, Peng YH, et al. Juvenile idiopathic arthritis with pulmonary hemosiderosis: a case report. $J$ Microbiol Immunol Infect 2002; 35: 133-5.

10. Le Clainche L, Le Bourgeois M, Fauroux B, et al. Long-term outcome of idiopathic pulmonary hemosiderosis in children. Medicine (Baltimore) 2000; 79: 318-26.

11. Cohen S. Idiopathic pulmonary hemosiderosis. Am J Med Sci 1999; 317: 67-74.

12. Cadnapaphornchai MA, Saulsbury FT, Norwood VF. Hypocomplementemic urticarial vasculitis: report of a pediatric case. Pediatr Nephrol 2000; 14: 328-31.

13. Black AK. Urticarial vasculitis. Clin Dermatol 1999; 17: $565-9$ 